\title{
Transtracheal aspiration using rigid bronchoscopy and a rigid needle for investigating mediastinal masses
}

\author{
M L Wilsher, A M Gurley
}

\begin{abstract}
Background - Use of the flexible needle via the fibreoptic bronchoscope to aspirate mediastinal nodes or masses has largely superseded the use of the rigid needle via the rigid bronchoscope. However, the yield at fibreoptic bronchoscopy is relatively low, although this improves with the use of a wider gauge needle. In this study the sensitivity and the safety of rigid needle sampling of the mediastinum in the diagnosis of lung cancer is evaluated.

Methods - Transtracheal needle aspiration (TTNA) was performed with the rigid bronchoscope and a rigid aspiration needle under general anaesthesia using a previous computed tomographic (CT) scan as a guide to the sample site. A cytopathologist immediately examined the specimens for adequacy and preliminary diagnosis, thus determining the number of aspirations.

Results - Twenty four patients were evaluated. The diagnostic sensitivity of TTNA was $88 \%$. This led to a management decision in 21 patients. There were no false positives and no complications.

Conclusions - TTNA using the rigid bronchoscope with CT scanning and a cytopathologist present is a sensitive and safe way of diagnosing lung cancer in patients with a mediastinal mass or enlarged mediastinal nodes.

(Thorax 1996;51:197-199)
\end{abstract}

Department of Respiratory Medicine $M$ L Wilsher

Department of Cytopathology A M Gurley

Green Lane Hospital, Auckland 3, New Zealand

Correspondence to: Dr M L Wilsher.

Received 6 December 1994 Returned to authors 21 February 1995 Revised version received 16 June 1995

Accepted for publication 29 September 1995
Keywords: bronchoscopy, cytology, lung cancer, mediastinum.

Transtracheal needle aspiration (TTNA) as a means of sampling mediastinal tissue was first described in $1958 .^{1}$ Using a rigid needle to puncture the main carina via the rigid bronchoscope a diagnostic sensitivity of $42 \%$ was achieved, and using an oesophageal varices needle Wang reported an improved sensitivity of $90 \% .^{2}$ In spite of this, the technique fell out of favour. With the development of a flexible needle which could be used via the fibreoptic bronchoscope, mediastinal lymph nodes could now be sampled at the same time as the initial diagnostic bronchoscopy. ${ }^{3}$ However, the positive yield using the flexible needle was low and other studies have since confirmed that the diagnostic sensitivity is around $50 \%$, although the use of a 19 gauge needle has improved this considerably. ${ }^{45}$

In our institution we found the initial experience with the flexible needle discouraging and returned to using the rigid needle via the rigid bronchoscope under general anaesthesia. We have recently reported that the presence of a cytopathologist in theatre to determine adequacy of the specimens increases the yield considerably. ${ }^{6}$

In this study we have prospectively evaluated the diagnostic sensitivity and safety of TTNA using such technique in 24 patients.

\section{Methods}

Twenty four consecutive patients in whom TTNA was performed using the rigid needle were evaluated prospectively. Patients were selected for the procedure if they were found to have a mediastinal mass or suspected malignant mediastinal lymphadenopathy on the computed tomographic (CT) scan. TTNA was performed in most patients for cytological diagnosis, the initial bronchoscopy being non-diagnostic, and in three as part of further staging where the diagnosis of non-small cell carcinoma was already secure.

\section{BRONCHOSCOPIC TECHNIQUE}

Rigid bronchoscopy under general anaesthesia was performed by experienced operators and, after inspection of the tracheobronchial tree, aspirates were taken from sites predetermined by evaluation of the CT scan. The rigid needle was advanced through the tracheal or bronchial wall and suction applied. The needle was moved back and forth with suction still applied, care being taken not to pull the needle out completely. Suction was discontinued before withdrawal. The rigid needles are either straight or angled allowing penetration into the tracheal wall and the length of the needle tip is $2 \mathrm{~cm}$, although this was rarely advanced to its full length (figure). The needles are stainless steel and they are sterilised and reusable. 


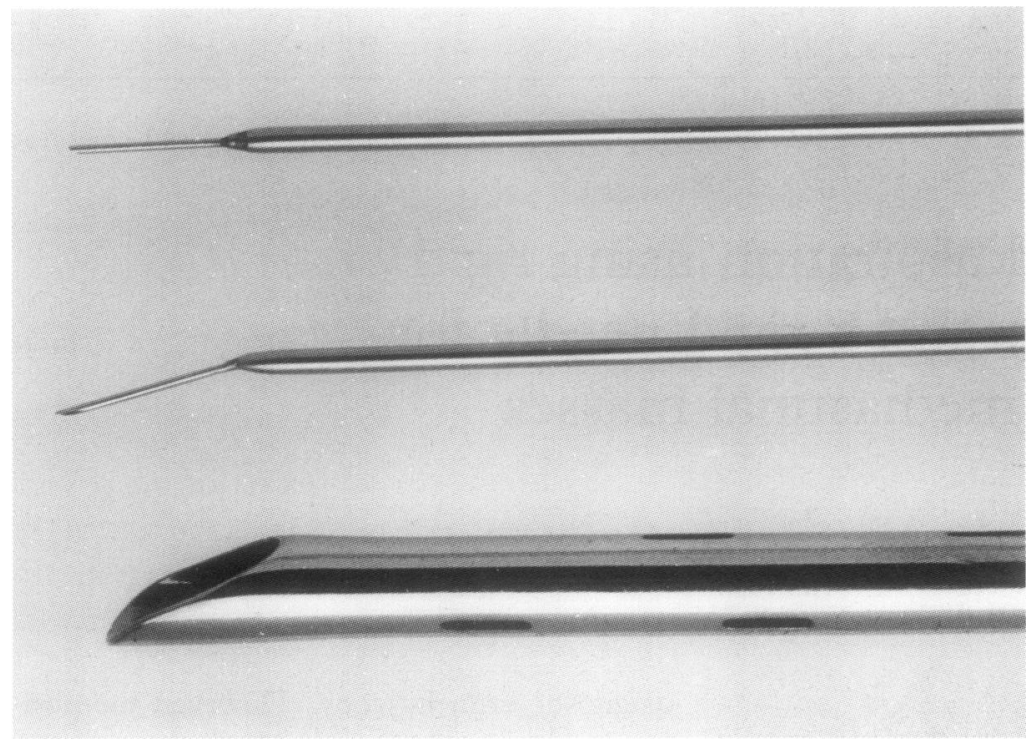

Rigid needles used for transtracheal needle biopsy shown alongside the rigid bronchoscope. in three, and extrinsic airway compression in 20.

TTNA was performed at one or more sites as follows: tracheal wall (14), main carina (three), right upper lung carina (three), left main bronchus (two), right main bronchus (three). The average number of aspirates taken was six (range 1-19). There were no complications and the time spent under anaesthesia was generally less than 20 minutes.

In 16 patients tissue from non-small cell carcinoma (adeno, squamous, or large cell undifferentiated) was aspirated and in two cases of small cell carcinoma. The remaining three in whom a diagnostic aspirate was achieved had metastatic melanoma, mucoepidermoid carcinoma, and non-caseating granulomas (presumed sarcoidosis), respectively. In all cases of malignancy the cytopathologist was able to indicate this as the likely diagnosis at the time of initial examination in theatre. Examination of the cell block subsequently confirmed these diagnoses. There were three patients in whom the aspirates were false negative. For two of these patients the samples contained necrotic tissue suggesting that the needle had been within tumour. These three patients were subsequently identified as having recurrent squamous cell carcinoma (previous lobectomy), metastatic breast carcinoma (as judged by clinical course), and small cell carcinoma (mediastinoscopy). There were no false positives during a follow up period of at least six months in all patients.

The sensitivity of TTNA was $88 \%$ and the specificity $100 \%$. Thus, TTNA led to a primary cytological diagnosis in 18 patients in whom no diagnosis had been achieved with previous investigations including fibreoptic bronchoscopy and, in one patient, additional percutaneous fine needle aspiration. It also confirmed N2 disease in three patients in whom the histological diagnosis of lung cancer had been made at earlier bronchoscopy. In this group of 21 patients management decisions were made based on the information gained from TTNA, and mediastinoscopy/mediastinotomy was avoided. specificity was defined as: true positives/true positives plus false positives (\%).

The test was considered positive if the diagnosis was benign or malignant neoplasm or benign abnormality. Negative test results included no abnormality, inconclusive, or no reading. Needle aspirates were considered false negative if the patient was subsequently found to have malignant mediastinal disease at mediastinoscopy or at thoracotomy, or the subsequent clinical course was consistent with mediastinal malignancy.

\section{Results}

A total of 24 procedures were performed on 24 patients ( 15 men) of mean age $56(31-75)$ years. Two patients had a normal chest radiograph but all had evidence of a mediastinal mass or nodes on the CT scan of the thorax. At initial fibreoptic bronchoscopy the macroscopic findings were: normal in two, endobronchial tumour in two, submucosal tumour infiltration

\section{Discussion}

TTNA performed with a rigid needle via the rigid bronchoscope is a safe and relatively easy procedure in experienced hands and can be performed as a day case. The presence of a cytopathologist in theatre to examine the adequacy of specimens and to provide a preliminary diagnosis, and the availability of a CT scan to determine sampling sites has improved the sensitivity of the test in our institution to $88 \%$. As such, TTNA is a useful adjunct in the diagnosis and, in selected patients, the staging of lung cancer. However, mediastinoscopy remains the investigation of choice if sampling is negative.

Since it was first described, TTNA using the rigid needle has resulted in a high diagnostic yield, but use has been limited by the need for operator experience with rigid bronchoscopy. The flexible bronchoscopic needle introduced 
by Wang therefore rapidly gained popularity. ${ }^{3}$ Although the sensitivity of this technique was initially reported by Wang as $76 \%,{ }^{4}$ we and others found the needle difficult to lodge into the mediastinum and this is reflected in subsequent reports of variable sensitivity in the diagnosis of lung and mediastinal malignancy. ${ }^{7-10}$ Since the early descriptions of the use of a flexible needle Wang has devised both an 18 gauge and 19 gauge needle. Overall, sensitivity using the Wang 18 gauge needle increased to $80 \%$ and with the 19 gauge needle to $89 \% .{ }^{511}$ Both needles provide histological material for diagnosis. It is noteworthy that in these latter two studies CT staging was employed and so the improved sensitivity may in part have been due to better selection of the sample sites.

The presence of a cytopathologist in theatre has had a major impact on the sensitivity of TTNA in our institution with an improvement from $18.8 \%$ in an earlier study ${ }^{6}$ to $81 \%$. The mean number of aspirates per procedure has increased but without complication. This study has shown that the sensitivity of TTNA has increased still further when a preoperative CT scan is available to guide the operator to the best site for sampling. TTNA using the flexible needle is safe with only a few reports of complications and rare false positive aspirates. ${ }^{12-14}$
We have experienced no major complications with the rigid needle in this institution.

We thank the physicians and bronchoscopy staff who allowed us to use their patient records and Miss M McKinlay for typing the manuscript.

Schieppati E. Mediastinal lymph node puncture through the tracheal carina. Surg Gynecol Obstet 1958;110:243-6.

2 Wang KP, Terry P, Marsh B. Bronchoscopic needle aspiration biopsy of paratracheal tumours. Am Rev Respir Dis 1978;118:17-21.

3 Wang KP, Terry P. Transbronchial needle aspiration in the diagnosis and staging of bronchogenic carcinoma. Am Rev diagnosis and staging of bro

4 Wang KP, Brower R, Haponik EF, Siegleman S. Flexible transbronchial needle aspiration for staging of brontransbronchial needle aspiration for stagin

5 Schenk DA, Chambers SL, Derdak S, et al. Comparison of the Wang 19-gauge and 22-gauge needle in the mediastinal staging of lung cancer. Am Rev Respir Dis 1993;147: staging

6 Roche DH, Wilsher ML, Gurley AM. Transtracheal needle aspiration. Diagn Cytopathol 1995;12:106-12.

7 Shure D, Fedullo PF. The role of transcarinal needle aspiration in the staging of bronchogenic carcinoma. Chest 1984;86:693-6.

8 Schenk D, Bryan CL, Bower JH, Myers DL. Transbronchial needle aspiration in the diagnosis of bronchogenic carcinoma. Chest 1987;92:83-5.

9 Wagner ED, Ramzy I, Greenberg D, Gonzales JM. Transbronchial fine-needle aspiration. Reliability and limitations. Am F Clin Pathol 1989;92:36-41.

10 Harrow EM, Oldenburg FA, Lingenfelter MS, Smith AM. Transbronchial needle aspiration in clinical practice. Chest 1989;96:1268-72.

11 Schenk DA, Strollo PJ, Pickard JS, et al. Utility of the Wang 19-gauge transbronchial histology needle in the staging of bronchogenic carcinoma. Chest 1989;96:272-4.

12 Kucera RF, Wolfe GK, Perry ME. Hemimediastinum after transbronchial needle aspiration. Chest 1986;90:466.

13 Sherling BE. Complication with a transbronchial histology needle Chest 1990:98:783-4.

14 Carlin BW, Hartell JH, Fedullo PF. False positive transcarinal needle aspirate in the evaluation of bronchogenic carcinoma. Am Rev Respir Dis 1989;140:1800-2.

\section{Angiotensin converting enzyme (ACE) inhibitor-induced cough and substance $P$}

Masafumi Tomaki, Masakazu Ichinose, Motohiko Miura, Yoshitaka Hirayama, Natsuko Kageyama, Hideyuki Yamauchi, Kunio Shirato

First Department of Internal Medicine, Tohoku University School of Medicine, 1-1 Seiryo-machi, Aoba-ku,

Sendai 980-77, Japan

M Tomaki

M Ichinose

M Miura

N Kageyama

$\mathrm{H}$ Yamauchi

K Shirato

Fujisawa

Pharmaceutical Co

Ltd, Osaka, Japan

Y Hirayama

Correspondence to: Dr K Shirato.

Received 9 January 1995

Returned to authors

23 March 1995

23 March 1995

8 September 1995

8 September 1995

Accepted for pub
9 October 1995
Abstract

Background - Angiotensin converting enzyme (ACE) inhibitors cause coughing in $5-10 \%$ of patients, but the exact mechanisms of this effect are still unclear. In the airways ACE degrades substance $P$ so the cough mechanism may be related to this peptide.

Methods - Nine patients who developed a cough and five patients who did not develop a cough when taking the $A C E$ inhibitor enalapril $(2.5$ or $5 \cdot 0 \mathrm{mg} / \mathrm{day})$ for hypertension were enrolled in the study. No subjects had respiratory disease and the respiratory function of all subjects was normal. One month after stopping enalapril, inhalation of hypertonic saline (4\%) was performed using an ultrasonic nebuliser for 15-30 minutes to induce sputum. The concentration of substance $P$ in the sputum sample was measured by radioimmunoassay. In four of the nine cases with a cough enalapril was given again for 1-2 weeks and the concentration of substance $P$ in the induced sputum was again measured.

Results - One month after stopping enalapril the mean (SE) concentration of substance $P$ in the sputum of the group with a cough was $16.6(3 \cdot 0) \mathrm{fmol} / \mathrm{ml}$, significantly higher than that in the subjects without a cough $(0.9(0.5) \mathrm{fmol} / \mathrm{ml})$. All four subjects in the group with a cough who were given a repeat dose of enalapril developed a cough again, but the concentrations of substance $P$ in the induced sputum while taking enalapril (17.9 $(3 \cdot 2) \mathrm{fmol} / \mathrm{ml}$ ) were similar to the values whilst off enalapril $(20 \cdot 0(2 \cdot 5) \mathrm{fmol} / \mathrm{ml})$. Conclusions - The mechanisms of ACE inhibitor-induced coughing may involve substance $\mathbf{P}$ mediated airway priming. 